\title{
Coumarins from Malaysian Micromelum minutum
}

\begin{abstract}
In a continuation of our study of the Rutaceae, detailed chemical investigation on Micromelum minutum (Rutaceae) collected from Sepilok, Sabah, Malaysia gave four new coumarins. The structures of the coumarins have been fully characterised by spectroscopic methods as 3nj4njdihydrocapnolactone 1, 2NjAjpoxyisocapnolactone 2, 8hydroxyisocapnolactone-2NB̈ Algiol 3 and 8-hydroxy-3nj4njdihydrocapnolactone-2N,B Aligiol 4.

Keyword: Micromelum minutum; Rutaceae; Coumarins; 3nj4njDihydrocapnolactone; $2 \mathrm{~N}, \mathrm{Nj}$ Epoxyisocapnolactone; 8-Hydroxyisocapnolactone-2 Nij Aliol; $\quad$ 8-Hydroxy-3nj4nj dihydrocapnolactone-2N,B Algiol
\end{abstract}

